\title{
Closed-Form BER Analysis for Antenna Selection Using Orthogonal Space-Time Block Codes
}

\author{
Saeed Kaviani and Chintha Tellambura \\ Department of Electrical and Computer Engineering \\ University of Alberta, Edmonton, CANADA \\ Email: \{saeedk,chintha\}@ece.ualberta.ca
}

\begin{abstract}
In this paper, we multiple-input multiple-output (MIMO) systems employing transmit antenna selection and orthogonal space-time block codes (OSTBCs) are not available. We thus derive exact closed-form expressions for the BER of Graycoded $M$-ary one and two-dimensional amplitude modulations when an OSTBC is employed and $N$ transmit antennas out of total $L_{t}$ antennas are selected for transmission. We also derive tight closed-form approximate BER for M-PSK constellations. Our BER expressions are valid for a frequency-flat Rayleigh fading MIMO channel and can be evaluated without numerical integration methods.
\end{abstract}

\section{INTRODUCTION}

Multiple antennas for transmitting and/or receiving data effectively mitigates fading. Obtaining the benefits of multiple transmit antennas requires the use of special space-time signaling schemes such as orthogonal space-time block codes (OSTBCs), a class of easily decoded space-time codes that achieve full diversity order [1], [2]. OSTBCs exist only for certain numbers of transmit antennas, and this limits their potential application. Decoding of OSTBCs is equivalent to decoding a number of independent single-input single-output data streams.

Antenna selection has been treated in some previous publication from two approaches. The first approach, concentrates on the capacity maximization through antenna selection. The focus of the other approach is on the minimization of the error rate for practical systems. Recently, there has been increasing interest in the combination of antenna selection and space-time codes. Transmit antenna selection (TAS), where OSTBC signal matrices are transmitted over a selected subset of transmit antennas, is a practical technique for the realization of full diversity [3]. Although receive antenna selection is a well researched topic where various channel/correlation models have been comprehensively treated (see [4]-[7] among many others), analogous comprehensive results are limited for TAS; e.g. a general, exact closed-form bit error rate (BER) analysis of TAS is not available to date. Although the symbol error rate (SER) of TAS is derived in [8], the formulas require numerical methods. In [9], the SER of single TAS and receive generalized selection combining (GSC) is derived. Exact BER for selecting only two transmit antennas with BPSK signals using the Alamouti code is derived in [10], [11]. The method used in [10], [11] consists of finding the joint probability density function (pdf) of two largest SNRs and convolving them.
In this paper, we provide general, closed-form BER expressions for $M$-ary Pulse Amplitude Modulation (PAM) and $M$ ary Quadrature Amplitude Modulation (QAM) constellations, and an approximate BER expression for $M$-ary Phase Shift Keying (PSK) with arbitrary $N \geq 2$ TAS employing OSTBCs. The MGF of $N$ largest instantaneous signal-to-noise ratios (SNRs) for GSC in Nakagami fading is derived in [12]. Since TAS involves selecting the $N$ columns of the channel matrix with the largest Frobenius norms, the results of [12] can be utilized to the problem in hand. Our BER approximations correctly reveal the full diversity order of the system.

Notation: The Frobenius norm of matrix $\mathbf{A}$ is denoted by $\|\mathbf{A}\|_{F}$ and the Euclidean norm for vector $\mathbf{h}$ is $\|\mathbf{h}\|=\left(h_{1}^{2}+\cdots+h_{L_{r}}^{2}\right)^{1 / 2}$. A circularly symmetric complex Gaussian variable with mean $\mu$ and variance $\sigma^{2}$ is denoted by $z \sim \mathcal{C N}\left(\mu, \sigma^{2}\right)$

\section{SySTEM MODEL}

We consider a MIMO system in a Rayleigh fading environment with $L_{t}$ transmit and $L_{r}$ receive antennas. Channel state information (CSI) is perfectly available at the receiver. $N\left(N \leq L_{t}\right)$ transmit antennas out of $L_{t}$ are selected and activated for the transmission of OSTBC signal matrices, while the remaining transmit antennas are inactive. Let $\widetilde{\mathbf{H}} \in \mathcal{C}^{L_{r} \times N}$ be a submatrix of the channel matrix $\mathbf{H} \in \mathcal{C}^{L_{r} \times L_{t}} . \mathbf{H}=\left[h_{i j}\right]$ where $h_{i j} \sim \mathcal{C N}(0,1)$ is the channel gain between the $i$ th transmit and $j$ th receive antenna. $\widetilde{\mathbf{H}}$ consists of the channel gains for the $N$ selected transmit antennas and $L_{r}$ received antennas. Suppose that $\mathbf{h}_{j}\left(j=1,2, \ldots, L_{t}\right)$ are columns of the channel matrix $\mathbf{H}$. The columns are sorted according to their norms; Assume that $\left\|\mathbf{h}_{i_{1}}\right\| \geq \ldots \geq\left\|\mathbf{h}_{i_{L_{t}}}\right\|$ where $i_{k} \in\left\{1,2, \ldots, L_{t}\right\}$. Thus, $\widetilde{\mathbf{H}}$ is defined as

$$
\widetilde{\mathbf{H}}=\left[\mathbf{h}_{i_{1}} \mathbf{h}_{i_{2}} \cdots \mathbf{h}_{i_{N}}\right] .
$$

With this selection criterion, we maximize the total received signal power at the receiver. The received signals are expressed as

$$
\mathbf{Y}=\sqrt{\frac{E_{s}}{N}} \widetilde{\mathbf{H}} \mathbf{X}+\mathbf{V}
$$

where $\mathbf{Y} \in \mathcal{C}^{L_{r} \times T}$ is the complex received signal matrix and $\mathbf{X} \in \mathcal{C}^{N \times T}$ is the complex transmitted signal matrix, which is a member of an OSTBC [2], [13]. $\mathbf{V} \in \mathcal{C}^{L_{r} \times T}$ is the additive noise matrix with independent and identical distributed entries 
of $\mathcal{C N}\left(0, N_{0}\right)$. The coefficient $\sqrt{E_{s} / N}$ ensures that the total transmitted power in each channel use is $E_{s}$ and independent of number of transmit antennas.

Assume that $Q$ symbols $\left\{s_{1}, \ldots, s_{Q}\right\}$ with average energy equal to one, chosen from an $M$-PAM or $M$-QAM constellations, are transmitted by the transmission matrix $\mathbf{X}$. Since $T$ symbol periods are necessary to transmit $Q$ symbols, the symbol rate $R_{s}$ of the STBC is defined as $R_{s}=Q / T$.

When an OSTBC is used, the MIMO system is equivalent to $Q$ independent single input single output (SISO) systems defined as [2], [13]

$$
\tilde{s}_{q}=\sqrt{\frac{E_{s}}{N}}\left(\frac{1}{R_{s}}\|\widetilde{\mathbf{H}}\|_{F}^{2}\right) s_{q}+\nu_{q}, q=1, \ldots, Q
$$

where $\nu_{q} \sim \mathcal{C N}\left(0, \frac{1}{R_{s}}\|\widetilde{\mathbf{H}}\|_{F}^{2} N_{0}\right)$. We conclude that the achievable SNR per bit in $M$-ary constellation is

$$
\gamma_{b}(\rho)=\frac{E_{s}}{N_{0}} \frac{1}{R_{s} N \log _{2} M}\|\widetilde{\mathbf{H}}\|_{F}^{2}=c \rho\|\widetilde{\mathbf{H}}\|_{F}^{2}
$$

where $\rho=\frac{E_{s}}{N_{0}}$ is the SNR per channel and $c=$ $1 /\left(R_{s} N \log _{2} M\right)$. Therefore, the antenna selection criterion in (1), which selects $N$ transmit antennas, maximizes the instantaneous SNR and thereupon minimizes the error rate.

Let $\gamma_{k}=c \rho\left\|\mathbf{h}_{k}\right\|^{2}, k=1,2, \ldots, L_{t}$, are the scaled norms of the columns of $\mathbf{H}$. In a flat Rayleigh MIMO channel, $\gamma_{k}$ are independent identically distributed (i.i.d.) chi-squared variable with $2 L_{r}$ degrees of freedom and variance $L_{r} c \rho$. The pdf of $\gamma_{k}$ is given by [14]

$$
f_{\gamma_{k}}\left(\gamma_{k}\right)=\frac{\gamma_{k}^{L_{r}-1}}{(c \rho)^{L_{r}}\left(L_{r}-1\right) !} e^{-\gamma_{k} / c \rho}, \quad \gamma_{k} \geq 0
$$

and the cumulative density distribution (cdf) is given by [14]

$$
F_{\gamma_{k}}\left(\gamma_{k}\right)=1-e^{-\gamma_{k} / c \rho} \sum_{k=0}^{L_{r}-1} \frac{1}{k !}\left(\frac{\gamma_{k}}{c \rho}\right)^{k}, \quad \gamma_{k} \geq 0
$$

In transmit antenna selection (1), the best $N$ antennas with the largest $\gamma_{k}$ are selected. Thus, the received SNR per bit (4) can be written as

$$
\gamma_{b}=\sum_{k=1}^{N} \gamma_{(k)}
$$

where $\gamma_{(k)}=c \rho\left\|\mathbf{h}_{i_{k}}\right\|^{2}$. The MGF of $\gamma_{b}$ is given by [15]

$$
\begin{aligned}
\Phi_{\gamma_{b}}(s)= & \left(L_{t}-N\right)\left(\begin{array}{c}
L_{t} \\
N
\end{array}\right) \int_{0}^{\infty} f_{\gamma_{k}}(x)\left[F_{\gamma_{k}}(x)\right]^{L_{t}-N-1} \\
& \times\left[\int_{x}^{\infty} e^{-s t} f_{\gamma_{k}}(t) d t\right]^{N} d x \\
= & \left(L_{t}-N\right)\left(\begin{array}{c}
L_{t} \\
N
\end{array}\right) \int_{0}^{\infty} \frac{x^{L_{r}-1} e^{-x / c \rho}}{(c \rho)^{L_{r}}\left(L_{r}-1\right) !} \\
& \times\left(1-e^{-x / c \rho} \sum_{k=0}^{L_{r}-1} \frac{1}{k !}\left(\frac{x}{c \rho}\right)^{k}\right)^{L_{t}-N-1} \\
& \times\left(\frac{e^{-x\left(s+\frac{1}{c \rho}\right)}}{(c \rho)^{L_{r}}} \sum_{k=0}^{L_{r}-1} \frac{1}{k !} \cdot \frac{x^{k}}{\left(s+\frac{1}{c \rho}\right)^{L_{r}-k}}\right)^{N} d x
\end{aligned}
$$

this is simplified in [12] as

$$
\begin{aligned}
\Phi_{\gamma_{b}}(s)= & N\left(\begin{array}{c}
L_{t} \\
N
\end{array}\right) \frac{(c \rho)^{-L_{r} N}}{\Gamma\left(L_{r}\right)^{N}} \sum_{i_{1}, \ldots, i_{N-1}} a\left(L_{r} ; i_{1}, \ldots, i_{N-1}\right) \\
& \prod_{k=1}^{N-1} \frac{i_{k} !}{k^{i_{k}}} \sum_{j=0}^{L_{t}-N}\left(\begin{array}{c}
L_{t}-N \\
j
\end{array}\right)(-1)^{j} \\
& \times\left\{\sum_{n \in B}\left(\begin{array}{c}
j \\
n_{0}, \ldots, n_{L_{r}-1}
\end{array}\right) \frac{\left(c_{n j}+L_{r}-1\right) !}{(c \rho)^{c_{n j}} A_{n j}}\right. \\
& \left.\frac{1}{\left(\frac{1}{c \rho}+s\right)^{r+N-1}} \cdot \frac{1}{\left(\frac{1}{c \rho}(N+j)+N s\right)^{c_{n j}+L_{r}}}\right\}
\end{aligned}
$$

where $a\left(L_{r} ; i_{1}, \ldots, i_{N-1}\right)$ is the coefficient of $x_{1}^{i_{1}} \ldots x_{N-1}^{i_{N-1}}$ in expression

$$
\left(x_{1}+x_{2}+\cdots+x_{N}\right)^{L_{r}-1}\left(x_{2}+\cdots+x_{N}\right)^{L_{r}-1} \cdots x_{N-1}^{L_{r}-1}
$$

and $B$ is the set of all combinations of nonnegative integers of $n_{0}, n_{1}, \ldots, n_{L_{r}-1}$ such that $\sum_{k=0}^{L_{r}-1} n_{k}=j, c_{n j}=$ $\sum_{k=1}^{L_{r}-1} k n_{k}, A_{n j}=\prod_{k=2}^{L_{r}-1}(k !)^{n_{k}}$ and $r=\sum_{k=1}^{N-1} i_{k}$.

\section{BER ANALYSIS OF $M$-ARY CONSTELLATIONS}

\section{A. Exact BER for M-ary PAM}

We first derive the BER for $M$-ary PAM with antenna selection and OSTBCs using Gray mapping. In an AWGN channel, the exact BER of the $n$-th bit is given by [16]

$$
P_{M}^{\mathrm{AWGN}}(n ; \rho)=\frac{2}{M} \sum_{i=0}^{k_{n}} B_{i} \mathcal{Q}\left(D_{i} \sqrt{\gamma_{b}(\rho)}\right)
$$

where

$$
\begin{aligned}
k_{n} & =\left(1-\frac{1}{2^{n}}\right) M-1 \\
B_{i} & =(-1)^{\left\lfloor\frac{i .2^{n-1}}{M}\right\rfloor}\left(2^{n-1}-\left\lfloor\frac{i .2^{n-1}}{M}+\frac{1}{2}\right\rfloor\right) \\
D_{i} & =(2 i+1) \sqrt{\frac{6 \log _{2} M}{M^{2}-1}} .
\end{aligned}
$$

Thus, to obtain the average BER, we take the expectation with respect to the channel statistics:

$$
\begin{aligned}
& P_{M}(n ; \rho)=\frac{2}{M} \sum_{i=0}^{k_{n}} B_{i} \mathcal{E}_{\widetilde{\mathbf{H}}}\left[\mathcal{Q}\left(D_{i} \sqrt{\gamma_{b}(\rho)}\right)\right] \\
& =\frac{2}{M} \sum_{i=0}^{k_{n}} B_{i} \frac{1}{\pi} \int_{0}^{\pi / 2} \Phi_{\gamma_{b}}\left(-\frac{D_{i}^{2}}{2 \sin ^{2} \theta}\right) d \theta
\end{aligned}
$$

where $\mathcal{Q}(x)=\frac{1}{\pi} \int_{0}^{\pi / 2} \exp \left(-\frac{x^{2}}{2 \sin ^{2} \theta}\right) d \theta$.

The exact average BER of an OSTBC with $M$-PAM is therefore given by

$$
P_{M}(\rho)=\frac{1}{\log _{2} M} \sum_{n=1}^{\log _{2} M} P_{M}(n ; \rho) .
$$


Therefore, the BER can be obtained from (9) and (14) as

$$
\begin{gathered}
P_{M}(\rho)=\frac{2 N}{M \log _{2} M}\left(\begin{array}{c}
L_{t} \\
N
\end{array}\right) \frac{(c \rho)^{-L_{r} N}}{\left[\left(L_{r}-1\right) !\right]^{N}} \sum_{n=1}^{\log _{2} M} \sum_{i=0}^{k_{n}} B_{i} \\
\sum_{i_{1}, \ldots, i_{N-1}} a\left(L_{r} ; i_{1}, \ldots, i_{N-1}\right) \prod_{k=1}^{N-1} \frac{i_{k} !}{k^{i_{k}}} \sum_{j=0}^{L_{t}-N}\left(\begin{array}{c}
L_{t}-N \\
j
\end{array}\right)(-1)^{j} \\
\times\left\{\sum_{n \in B}\left(\begin{array}{c}
j \\
n_{0}, \ldots, n_{L_{r}-1}
\end{array}\right) \frac{\left(c_{n j}+L_{r}-1\right) !}{(c \rho)^{2 c_{n j}+L_{r}+r+N-1} A_{n j}}\right. \\
\left.\quad \times I\left(\frac{c \rho D_{i}^{2}}{2}, \frac{N c \rho D_{i}^{2}}{(N+j)} ; r+N-1, c_{n j}+L_{r}\right)\right\} .
\end{gathered}
$$

and

$$
I\left(c_{1}, c_{2} ; m_{1}, m_{2}\right)=\frac{1}{\pi} \int_{0}^{\pi / 2}\left(\frac{\sin ^{2} \theta}{\sin ^{2} \theta+c_{1}}\right)^{m_{1}}\left(\frac{\sin ^{2} \theta}{\sin ^{2} \theta+c_{2}}\right)^{m_{2}} d \theta
$$

is given in [17, pp. 162].

\section{B. Exact BER for $M$-ary $Q A M$}

Note that a rectangular or square QAM constellations can be composed to two independent PAM constellations: $I$-ary PAM for the in-phase component and $J$-ary PAM for the quadrature component, where $M=I \times J$. Thus, the exact average BER of $M$-QAM is given by

$$
P_{M}(\rho)=\frac{1}{\log _{2}(I . J)}\left(\sum_{n=1}^{\log _{2} I} P_{I}(n ; \rho)+\sum_{m=1}^{\log _{2} J} P_{J}(m ; \rho)\right) .
$$

The result for transmit diversity and one receiver antenna $\left(L_{r}=1\right)$ can be simplified as

$$
\Phi_{\gamma_{b}}(s)=\frac{L_{t} !}{(N-1) !} \frac{1}{(1+c \rho s)^{N-1}} \prod_{j=0}^{L_{t}-N} \frac{1}{N(1+c \rho s)+j}
$$

where we have used $\sum_{j=0}^{n}\left(\begin{array}{c}n \\ j\end{array}\right) \frac{(-1)^{j}}{p+j}=\frac{n !}{p(p+1) \cdots(p+n)}$ to convert the sum to the product (19). Considering that

$$
\begin{aligned}
& \lim _{\rho \rightarrow \infty} P_{M}(\rho) \rho^{L_{t}}=\frac{2}{M \log _{2} M}\left(\sum_{n=1}^{\log _{2} M} \sum_{i=0}^{k_{n}} \frac{B_{i}}{D_{i}^{2 L_{t}}}\right) \\
& \times \frac{1}{N^{L_{t}-N+1} c^{L_{t}}} \cdot \frac{\left(2 L_{t}-1\right) !}{2^{L_{t}-1}\left(L_{t}-1\right) !(N-1) !}, \quad \rho \gg 1
\end{aligned}
$$

we can approximate (16) as

$$
\begin{aligned}
P_{M}(\rho) & \approx \frac{2}{M \log _{2} M}\left(\sum_{n=1}^{\log _{2} M} \sum_{i=0}^{k_{n}} \frac{B_{i}}{D_{i}^{2 L_{t}}}\right) \frac{1}{N^{L_{t}-N+1} c^{L_{t}}} \\
& \times \frac{\left(2 L_{t}-1\right) !}{2^{L_{t}-1}\left(L_{t}-1\right) !(N-1) !}\left(\frac{1}{\rho}\right)^{L_{t}}, \quad \rho \gg 1
\end{aligned}
$$

which clearly indicates a full diversity order of $L_{t}$ at high SNRs for $N$ transmit antenna selection of an $L_{t} \times 1$ system.

\section{Approximate BER for M-ary PSK}

A tight approximation for the BER of the coherent $M$-ary PSK in AWGN channels is given by [18]

$$
\begin{aligned}
P_{M}^{\mathrm{AWGN}}(\rho) & \simeq \frac{2}{\max \left(\log _{2} M, 2\right)} \\
\times & \sum_{i=1}^{\max (M / 4,1)} \mathcal{Q}\left(\sqrt{2 \sin ^{2} \frac{(2 i-1) \pi}{M} \gamma_{b}(\rho)}\right) .
\end{aligned}
$$

Again, using the expression for $\mathcal{Q}$-function and same derivation steps from (14) to (16)

$$
\begin{aligned}
P_{M}(\rho)= & \mathcal{E}_{\tilde{\mathbf{H}}}\left[P_{M}^{\mathrm{AWGN}}(\rho)\right] \simeq \frac{2}{\max \left(\log _{2} M, 2\right)} \\
& \times \sum_{i=1}^{\max (M / 4,1)} \mathcal{E}_{\tilde{\mathbf{H}}}\left[\mathcal{Q}\left(\sqrt{2 \sin ^{2} \frac{(2 i-1) \pi}{M} \gamma_{b}(\rho)}\right)\right] \\
& =\frac{2}{\max \left(\log _{2} M, 2\right)} \\
& \times \sum_{i=1}^{\max (M / 4,1)} \int_{0}^{\pi / 2} \Phi_{\gamma_{b}}\left(-\frac{\sin ^{2} \frac{(2 i-1) \pi}{M}}{\sin ^{2} \theta}\right) d \theta
\end{aligned}
$$

an approximate expression for the average BER of OSTBC can be found similarly as

$$
\begin{gathered}
P_{M}(\rho) \simeq \frac{2 N}{\max \left(\log _{2} M, 2\right)}\left(\begin{array}{c}
L_{t} \\
N
\end{array}\right) \frac{(c \rho)^{-L_{r} N}}{\left[\left(L_{r}-1\right) !\right]^{N}} \sum_{i=1}^{\max (M / 4,1)} \\
\sum_{i_{1}, \ldots, i_{N-1}} a\left(L_{r} ; i_{1}, \ldots, i_{N-1}\right) \prod_{k=1}^{N-1} \frac{i_{k} !}{k^{i_{k}}} \sum_{j=0}^{L_{t}-N}\left(\begin{array}{c}
L_{t}-N \\
j
\end{array}\right)(-1)^{j} \\
\times\left\{\sum_{n \in B}\left(\begin{array}{c}
j \\
n_{0}, \ldots, n_{L_{r}-1}
\end{array}\right) \frac{\left(c_{n j}+L_{r}-1\right) !}{(c \rho)^{2 c_{n j}+L_{r}+r+N-1} A_{n j}}\right. \\
\left.\times I\left(C_{i, 1}, C_{i, 2} ; r+N-1, c_{n j}+L_{r}\right)\right\}
\end{gathered}
$$

where

$$
\begin{gathered}
C_{i, 1}=c \rho \sin ^{2} \frac{(2 i-1) \pi}{M}, \\
C_{i, 2}=\frac{2 N c \rho}{(N+j)} \sin ^{2} \frac{(2 i-1) \pi}{M} .
\end{gathered}
$$

IV. Numerical RESUlts

In this section, we consider the orthogonal design proposed in [2], [13], namely the rate $3 / 4$ with $3 \leq L_{t} \leq 6$ transmit antennas. Thus, to use this orthogonal design, we need to select $N=3$ transmit antennas. Fig. 1 compares the exact expression (16), the approximation (21), and the Monte Carlo simulation results for the system with $L_{r}=1$ receive antennas, all using 16-QAM signal constellations. Note that (16) asymptotically approaches (21), which is a tight bound of (16) at high SNRs. Fig. 2 shows the exact BER for $\left(L_{t} ; N, L_{r}\right)$ systems, where OSTBC is tranmitted over $N$ selected antennas of $L_{t}$ available transmit antennas. $L_{r}$ is the number of receive antennas in the system. Again, 16-QAM signal constellation is used to show the general forms of BER expressions. 


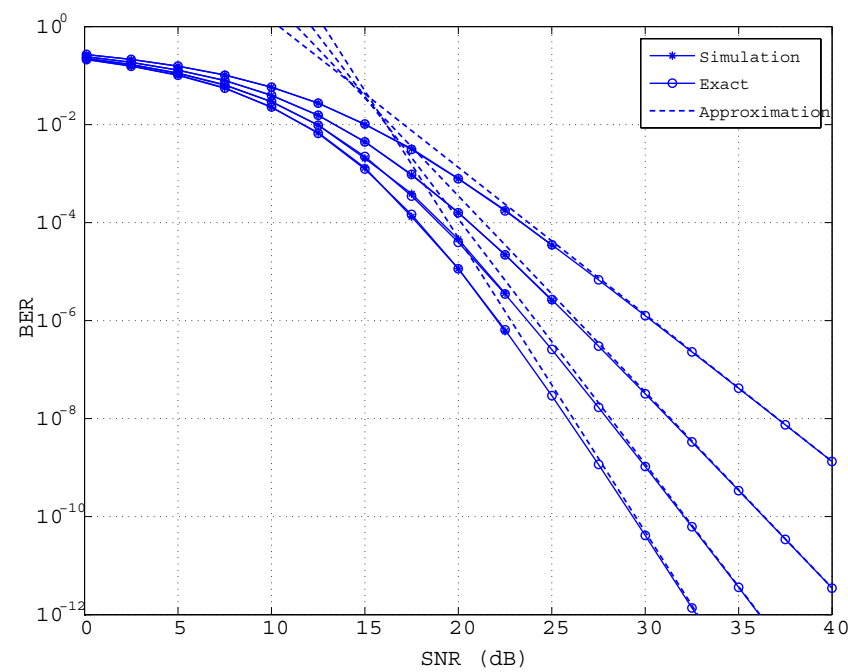

Fig. 1. Comparison between the exact expression, approximation and simulation for $N=3$ TAS out of $3 \leq L_{t} \leq 6$ with $L_{r}=1,16$-QAM.

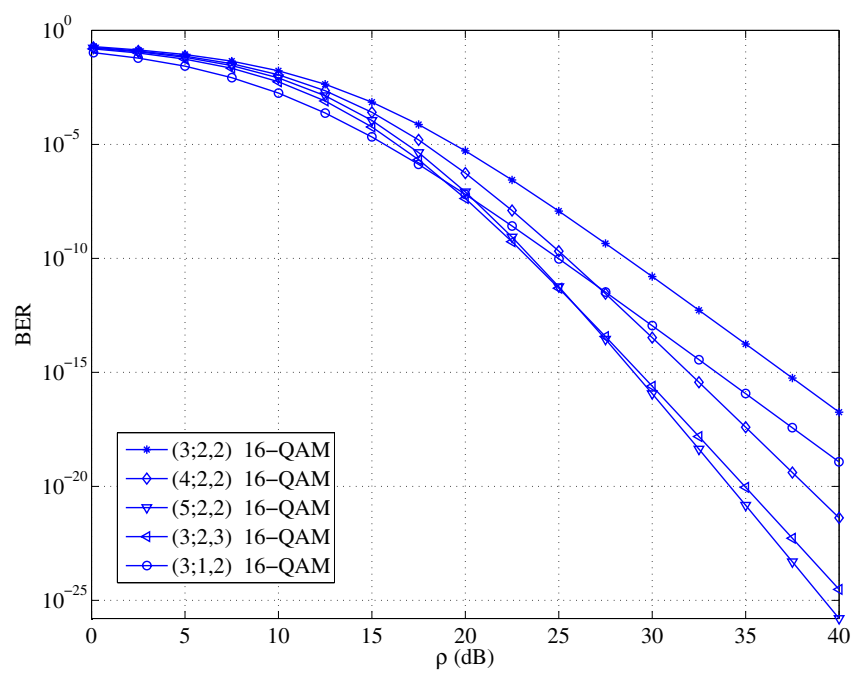

Fig. 2. Exact BER derived in (16) for $\left(L_{t} ; N, L_{r}\right)$ TAS systems, using 16-QAM

\section{CONCLUSION}

In this paper, we have investigated the performance of TAS and OSTBCs. The exact BER for M-PAM and M-QAM and an approximate BER for M-PSK were derived. Our results are sufficiently general to handle an arbitrary number of antennas, unlike the previous results. Moreover, we directly derived the BER, not via the symbol error probability. As expected, we find that this scheme achieves full diversity order asymptotically (i.e., $L_{t}$ not $N$ ), as if all the transmit antennas were used.

\section{REFERENCES}

[1] S. Alamouti, "A simple transmit diversity technique for wireless communications," IEEE J. Select. Areas Commun., vol. 16, pp. 1451-1458, Oct. 1998.

[2] V. Tarokh, H. Jafarkhani, and A. Calderbank, "Space-time block codes from orthogonal designs," IEEE Trans. Inform. Theory, vol. 45, pp. 1456-1467, July 1999.
[3] D. A. Gore and A. J. Paulraj, "MIMO antenna subset selection with space-time coding," IEEE Trans. Signal Processing, vol. 50, pp. 25802588, Oct. 2002.

[4] G. K. Karagiannidis, "Performance analysis of SIR-based dual selection diversity over correlated Nakagami-m fading channels," IEEE Trans. Veh. Technol., vol. 52, pp. 1207-1216, Sept. 2003.

[5] V. A. Aalo and T. Piboongungon, "On the multivariate generalized gamma distribution with exponential correlation," IEEE Global Telecommn. Conf. (GLOBECOM), vol. 3, pp. 1229-1233, Dec. 2005.

[6] Q. T. Zhang and H. G. Lu, "A general analytical approach to multibranch selection combining over various spatially correlated fading channels," IEEE Trans. Commun., vol. 50, pp. 1066-1073, July 2002.

[7] N. C. Sagias, G. K. Karagiannidis, D. A. Zogas, P. T. Mathiopoulos, and G. S. Tombras, "Performance analysis of dual selection diversity in correlated Weibull fading channels," IEEE Trans. Commun., vol. 52, pp. 1063-1067, July 2004.

[8] D. J. Love, "On the probability of error of antenna-subset selection with space-time block codes," IEEE Trans. Commun., vol. 53, pp. 1799-1803, Nov. 2005.

[9] X. Cai and G. B. Giannakis, "Performance analysis of combined transmit selection diversity and receive generalized selection combining in Rayleigh fading channels," IEEE Commun. Lett., vol. 3, pp. 19801983, Nov. 2004

[10] Z. Chen, J. Yuan, B. Vucetic, and Z. Zhou, "Performance of Alamouti scheme with transmit antenna selection," IEE Elect. Lett., vol. 39, pp. 1666-1668, Nov. 2003.

[11] _ "Performance of Alamouti scheme with transmit antenna selection," IEEE Int. Symposium on Personal, Indoor and Mobile Radio Commun. (PIMRC), vol. 2, pp. 1135-1141, Sept. 2004.

[12] Y. Ma and C. C. Chai, "Unified error probability analysis for generalized selection combining in Nakagami fading channels," IEEE J. Select. Areas Commun., vol. 18, pp. 2198-2210, Nov. 2000.

[13] E. G. Larsson and P. Stoica, Space-time Block Coding for Wireless Communications. Cambridge University Press, 2003.

[14] J. G. Proakis, Digital Communications, 4th ed. New York: McGraw Hill, 2000.

[15] A. Annamalai, G. K. Deora, and C. Tellambura, "Theoretical diversity improvement in $\operatorname{GSC}(\mathrm{N}, \mathrm{L})$ receiver with nonidentical fading statistics," IEEE Trans. Commun., vol. 53, pp. 1027-1035, June 2005.

[16] K. Cho and D. Yoon, "On the general BER expression of one- and twodimensional amplitude modulations," IEEE Trans. Commun., vol. 50, pp. 1074-1080, July 2002.

[17] M. K. Simon and M.-S. Alouini, Digital Communication over Fading Channels, 1st ed. New York: Wiley, 2000.

[18] J. Lu, K. B. Letaief, J. C. Chuang, and M. L. Liou, "M-PSK and M-QAM BER computation using signal-space concepts," IEEE Trans. Commun., vol. 47, pp. 181-184, Feb. 1999. 